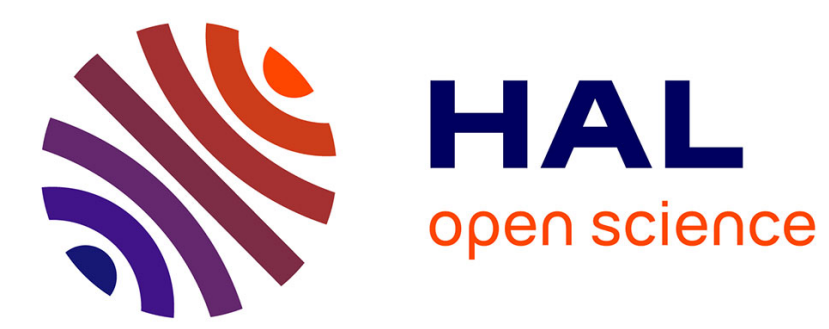

\title{
Le pari économique de la Chine sur l'OMC : réalités, mythes et inconnues, \\ Jean-François Huchet
}

\section{To cite this version:}

Jean-François Huchet. Le pari économique de la Chine sur l'OMC: réalités, mythes et inconnues,. Perspectives chinoises, 2002, 70, pp.4-14. 10.3406/perch.2002.2728 . hal-02543957

\section{HAL Id: hal-02543957 \\ https://hal.science/hal-02543957}

Submitted on 15 Apr 2020

HAL is a multi-disciplinary open access archive for the deposit and dissemination of scientific research documents, whether they are published or not. The documents may come from teaching and research institutions in France or abroad, or from public or private research centers.
L'archive ouverte pluridisciplinaire HAL, est destinée au dépôt et à la diffusion de documents scientifiques de niveau recherche, publiés ou non, émanant des établissements d'enseignement et de recherche français ou étrangers, des laboratoires publics ou privés. 


\section{Le pari économique de la Chine sur l'OMC : réalités, mythes et} inconnues

Jean-François Huchet

\section{Citer ce document / Cite this document :}

Huchet Jean-François. Le pari économique de la Chine sur l'OMC : réalités, mythes et inconnues. In: Perspectives chinoises, $\mathrm{n}^{\circ} 70$, 2002. pp. 4-14;

doi : https://doi.org/10.3406/perch.2002.2728

https://www.persee.fr/doc/perch_1021-9013_2002_num_70_1_2728

Fichier pdf généré le 24/04/2018 


\section{Le pari économique de la Chine sur l'OMC : réalités, mythes et inconnues}

\section{Jean-François Huchet}

$\mathrm{P}$ EU d'évènements auront déchaîné autant de passions, généré autant d'analyses et de publications, suscité autant d'espoirs ou d'inquiétudes au sein de la population chinoise que l'accession de la Chine à l'Organisation mondiale du commerce (OMC). L'enjeu il est vrai, du moins théoriquement, est considérable pour ce pays. Même si on laisse de côté l'orgueil national, le sentiment de ne plus être exclu de ce « club» - pourtant moins fermé qu'on le dit souvent - que constitue l'OMC, les modifications du régime du commerce extérieur que la Chine va devoir mettre en place tout comme les droits issus de son accession sont potentiellement porteurs de vastes conséquences économiques ${ }^{(1)}$. Mais c'est aussi parce que l'accession à l'OMC est considérée comme une nouvelle étape, un nouveau souffle, un catalyseur des réformes économiques internes, bref comme un instrument de politique intérieure. On comprend de ce fait l'ampleur comme la dramatisation des débats sur l'OMC que l'on pourrait comparer à ceux qui eurent lieu dans les sociétés espagnole, portugaise ou grecque lors de leur adhésion à la Communauté européenne dans les années 1980, ou à ceux qui se déroulent aujourd'hui dans les pays de l'Europe centrale et orientale qui négocient leur entrée dans l'Union européenne. Beaucoup d'analyses ont également comparé l'accession de la Chine à l'OMC au lancement de la politique d'ouverture en décembre 1978, c'est-à-dire une décision de politique économique générique qui devrait bouleverser les structures et les modes de fonctionnement de l'économie chi- noise. Comme en 1978, les dirigeants semblent en effet acculés à réformer et à produire une forte croissance économique dont ils tirent leur légitimité, sous peine d'être rattrapés par les problèmes structurels de l'économie chinoise.

Dans ce contexte, rien d'étonnant au fait de retrouver un spectre très large d'analyses sur les conséquences économiques de l'accession de la Chine à l'OMC. Entre les pessimistes qui insistent sur les incapacités de la Chine à relever des défis dont on grossit les traits pour mieux annoncer le chaos, et les optimistes béats qui voient dans l'OMC une fusée capable de placer en un temps record et sans encombre la Chine sur l'orbite des pays les plus développés sur le plan économique, cet article se propose d'apporter une analyse plus prudente et contrastée. Cette analyse insistera en particulier sur les facteurs qui risquent de limiter l'ouverture du marché intérieur comme sur les innombrables inconnues qui n'ont pas été élucidées par les modèles économétriques et qui sont susceptibles de réserver bien des surprises quant à l'influence de l'accession de la Chine à l'OMC sur le développement économique de ce pays.

\section{Un choc externe amorti}

Parmi les interrogations soulevées par bon nombre d'analyses sur les risques encourus par la Chine du fait de la modification de son régime de commerce extérieur, figure en première place la crainte qu'une ouverture trop importante et trop rapide du marché intérieur ne déstabilise durablement les producteurs nationaux. Un examen plus attentif des conditions de ventes des 
produits importés, des stratégies à long terme choisies par les investisseurs étrangers ainsi que des conditions d'application des règles de l'OMC permet néanmoins de tempérer très fortement cette anticipation négative. En effet, de nombreux « facteurs amortisseurs » (buffering effects ${ }^{(2)}$ ) devraient tempérer le choc de l'ouverture pour les producteurs nationaux.

\section{Ouverture négociée et effets " amortisseurs" : l'exemple de l'agriculture}

Des prix intérieurs chinois trop éloignés des prix mondiaux conjugués à la baisse des tarifs douaniers prévue dans l'accord OMC pourraient, selon certaines analyses, provoquer un basculement de la demande intérieure vers les produits importés et une chute drastique du chiffre d'affaires des producteurs nationaux, accélérant ainsi un exode rural massif vers les villes côtières. Cette possibilité a été très largement évoquée au sujet des produits agricoles de base notamment du blé, du soja, du riz et tout particulièrement pour les producteurs des régions les plus pauvres aux coûts de production les plus élevés et qui sont par conséquent plus exposés que les producteurs des régions côtières. Plusieurs facteurs incitent nćanmoins à relativiser le choc attendu en matière agricole :

- Les accords signés par la Chine pour son accession à l'OMC prévoient des garanties en matière de protection de son marché intérieur agricole. Des quotas d'importation maximums ont été négociés sur la base des accords généraux prévus dans l'Uruguay Round pour la libéralisation des produits agricoles. Les quotas d'accès au marché intérieur pour les importations sont déterminés par rapport à la consommation intérieure. Ces quotas seront progressivement revus à la hausse sur une base annuelle jusqu'en 2004 comme le montre le tableau $1^{(3)}$. Bien que, comme le précise Nicholas Lardy, la Chine ait accepté des quotas d'importations relativement plus importants que d'autres pays membres de l'OMC, le tableau 1 montre que les quotas d'importations maximums représentent une part relativement faible de la consommation intérieure et cela même si, en raison de la taille du marché chinois, les importations sont significatives à l'échelle mondiale ${ }^{(4)}$. Par ailleurs, en cas de dépassement des quotas, la Chine peut imposer des droits de douane capables de protéger de manière efficace son marché intérieur (dernière colonne du tableau 1), même si elle a accepté d'appliquer des droits de douane inférieurs à ceux maintenus par de nombreux autres membres de l'OMC. Ces quotas devraient ainsi limiter l'effet sur les producteurs locaux.

- Par ailleurs, en dépit des variations observées entre les différentes catégories de produits agricoles, les prix mondiaux et les prix chinois convergent déjà de plus en plus depuis plusieurs années grâce aux gains de productivité réalisés dans l'agriculture chinoise. Cette tendance devrait à l'avenir améliorer la compétitivité des produits chinois par rapport aux importations.

- Enfin, le marché intérieur chinois est loin d'être intégré. Les coûts de transport des produits agricoles sont, en moyenne, cinq fois supérieurs à ceux enregistrés aux Etats-Unis. Les producteurs des régions intérieures qui vendent sur les marchés locaux ne devraient donc pas subir une concurrence instantanée. En effet, le coût du transport des produits importés vers ces marchés renchérit sensiblement leur prix ${ }^{(5)}$. Ce phénomène devrait donc, encore pendant quelques années, permettre aux paysans les plus pauvres dans les régions reculées de diversifier leur production ou de tenter d'accroître leur productivité.

\section{Tableau 1 Exemple d'évolution des quotas d'importation et droits de douane sur les produits agricoles}

\begin{tabular}{|c|c|c|c|c|c|c|}
\hline Produits agricoles & $\begin{array}{c}\text { Importations } \\
1998 \\
\text { (millions } \\
\text { de tonnes) }\end{array}$ & $\begin{array}{l}\text { Quotas initiaux } \\
\text { d'importation } \\
\text { (2002) } \\
\text { (millions } \\
\text { de tonnes) }\end{array}$ & $\begin{array}{c}\text { Quotas fin de } \\
\text { période (2004) } \\
\text { (millions } \\
\text { de tonnes) }\end{array}$ & $\begin{array}{c}\% \text { de la } \\
\text { consommation } \\
\text { intérieure en } \\
\text { début et fin } \\
\text { de période } \\
(2002-2004)\end{array}$ & $\begin{array}{c}\text { Droits de } \\
\text { douane sur } \\
\text { les } \\
\text { importations } \\
\text { inférieures aux } \\
\text { quotas }\end{array}$ & $\begin{array}{l}\text { Droits de douane sur } \\
\text { les importations } \\
\text { supérieures aux } \\
\text { quotas début et fin de } \\
\text { période (2002-2004, } \\
\text { sauf * 2006) }\end{array}$ \\
\hline Blé & 1,55 & 7,30 & 9,30 & $6,1 \%-7,8 \%$ & $1 \%$ & $80 \%-43 \%$ \\
\hline Maïs & 0,25 & 4,50 & 7,20 & $4,7 \%-6,4 \%$ & $1 \%$ & $80 \%-43 \%$ \\
\hline Riz & 0,26 & 2,66 & 5,32 & $1,4 \%-2,7 \%$ & $1 \%$ & $80 \%-43 \%$ \\
\hline Huile de soja & 0,83 & 2,50 & 3,60 & - & $9 \%$ & $85 \%-9 \% *$ \\
\hline Coton & 0,21 & 0,743 & 0,894 & - & $1 \%$ & $76 \%-40 \%$ \\
\hline
\end{tabular}


Les coûts de sortie des investisseurs étrangers : exemple de l'automobile

Limpact sur le développement de l'industrie nationale a été largement évoqué comme un des principaux éléments négatifs de l'accession de la Chine à l'OMC. Les modèles économétriques, notamment ceux développés par Yang, pour la Banque mondiale, ou bien encore celui de Li Shantong et Zhai Fai du Centre de développement du Conseil des affaires de l'Etat prévoient que certaines industries comme l'automobile ou les machines-outils devraient souffrir de l'accession de la Chine à l'OMC en termes d'emplois et de production ${ }^{(6)}$. En sera-t-il vraiment ainsi ? La capacité de la Chine à développer des constructeurs chinois sans contrôle étranger majoritaire est certes gravement compromise aujourd'hui. Mais les conséquences en matière d'emplois et de production sont loin d'avoir été entièrement anticipées par les différents modèles économétriques.

Tout d'abord, avec l'augmentation de la consommation attendue de la baisse des prix, même en imaginant qu'une grande partie des voitures achetées soit importées, les activités de services (distribution, réparation, marché d'occasion) vont se développer rapidement, entrainant une hausse de l'emploi dans ces secteurs. Par ailleurs, d'importants segments de l'industrie sont contrôlés par des firmes chinoises (véhicules utilitaires, camions, autobus), et sont très compétitifs par rapport à la concurrence étrangère (le groupe Dongfeng, ou le groupe automobile $n^{\circ} 1$ Yiqi). Enfin, une analyse de la situation des constructeurs automobiles étrangers qui, organisés en joint ventures, dominent actuellement la production en Chine, indique que ceux-ci ne vont pas se retirer du marché chinois. Ces entreprises sino-étrangères vivaient jusqu'à aujourd'hui à l'abri de protections douanières qui leur permettaient de pratiquer des tarifs très largement au-dessus des prix mondiaux et de ne pas être soumis à la course technologique ainsi qu'à l'introduction annuelle de nouveaux modèles. Depuis l'accession de la Chine à l'OMC, tout indique que ces entreprises vont adapter leur stratégie plutôt que de renoncer à leur implantation. Les coûts de sortie pour les producteurs d'automobiles étrangers qui souhaiteraient abandonner la Chine seraient en effet d'autant plus considérables que ceux-ci ont investis dans des outils de production surdimensionnés (Citroën pourraient fabriquer chaque année 150000 voitures, alors qu'aujourd'hui les ventes sont de l'ordre de 50000 véhicules). Sachant que le marché chinois va progressivement se développer, ces constructeurs vont simplement procéder comme dans les autres parties du monde ouvertes à la concurrence internationale. Cette adaptation va passer par une baisse des prix de leurs véhicules (qui a déjà commencé) et l'introduction de nouveaux modèles avec une fré- quence plus rapide, ce qui implique une délocalisation d'une partie des activités de recherche et développement. Enfin, les constructeurs étrangers vont chercher à intégrer les usines implantées en Chine dans le réseau régional asiatique de production (peut être même pour certaines faire de l'usine chinoise une des plaques tournantes du réseau asiatique) ${ }^{(7)}$. Pour toutes ces raisons, même si la probabilité de voir survivre un producteur entièrement chinois est faible (à l'image de l'Angleterre), en termes d'emplois et de production, l'industrie automobile telle qu'elle existe aujourd'hui en Chine ne devrait pas sortir obligatoirement perdante de l'accession à l'OMC.

\section{Régimes douaniers d'exception et contrebande}

Les concessions accordées par la Chine sont conséquentes au regard de son ancien régime de commerce extérieur et des avantages dont bénéficient les autres pays en développement membres de l'OMC. Néanmoins, beaucoup d'exceptions au régime général avaient été négociées avant l'accession de la Chine à l'OMC, par différents membres sur plusieurs catégories de produits. La baisse des droits de douane prévue dans le protocole d'accession aura donc pour beaucoup de firmes industrielles une incidence relativement limitée. De surcroit, le commerce de contrebande ou le commerce gris avait atteint ces dernières années une telle importance pour les entreprises chinoises comme pour les entreprises étrangères installées en Chine, notamment dans les zones littorales, que les tarifs douaniers étaient inopérants pour une partie non négligeable des importations, voire même pour certains produits de consommation. Les innombrables scandales autour de la contrebande qui ont éclaté au cours de ces dernières années en témoignent. La campagne de lutte contre la contrebande, en 1998, a permis d'obtenir quelques chiffres révélateurs de l'ampleur du phénomène. Les douanes chinoises ont estimé que près de 100 milliards de yuans d'importations illégales, soit $10 \%$ du total des importations, étaient entrées en 1997 sur le territoire chinois ${ }^{(8)}$. Pour certains produits, tels les circuits intégrés, les aciers spéciaux, les automobiles, les cigarettes, les films photographiques, le pétrole, les proportions sont considérables. Dans le domaine des aciers spéciaux par exemple, les importations illégales représentaient en 1997, 200\% de la production nationale ${ }^{(4)}$. Dans le secteur des tubes cathodiques pour téléviseurs, le succès de la campagne sur les importations illégales a provoqué un triplement des importations légales ${ }^{i 11 !}$. Dans l'automobile, un rapport de l'Institut chinois de recherche sur le développement de l'industrie automobile estimait que les importations illégales représentaient près de $17 \%$ de la demande intérieure " $"$. Tous ces 
éléments incitent donc à relativiser l'impact réel des concessions accordées par la Chine en matière de baisse des droits de douane.

\section{Flexibilités de l'application des règles de l'OMC et échappatoires}

Un autre thème largement évoqué à propos des risques encourus par la Chine en matière d'ouverture de son marché intérieur est celui de la stricte application des règles de l'OMC. Non seulement la Chine aurait négocié des règles plus contraignantes que celles normalement appliquées aux autres membres de l'OMC, mais elle disposerait de très peu de marges de manœuvre dans leur application ${ }^{(12)}$. Le protocole d'accession de la Chine à l'OMC prévoit, il est vrai, des mécanismes de surveillance visant à vérifier l'application des concessions que la Chine a accordées à ses principaux partenaires commerciaux ${ }^{(13)}$. Néanmoins, plusieurs facteurs pourraient lui permettre d'appliquer de manière plus flexible ces règles, et même lui fournir des échappatoires.

Le processus de règlement des litiges commerciaux devant l'Organe de règlement des différents (ORD) de l'OMC ne relève pas uniquement de principes issus du droit $\mathrm{OMC}$, mais dépend également de négociations diplomatiques entre les Etats ${ }^{\prime 4}$. En imaginant qu'une entreprise française soit victime d'une non-application par la Chine d'une règle de l'OMC acceptée par ce pays, elle doit se lancer dans un véritable parcours du combattant. Elle doit dans un premier temps constituer un dossier visant à démontrer le préjudice subi, dossier qu'elle remet auprès de son ambassade qui va le transmettre à ses autorités de tutelle en France. Ensuite, celles-ci vont communiquer le dossier à la Commission de l'Union européenne à Bruxelles qui seule est habilitée à engager une procédure devant l'ORD. Puis s'engage à ce stade une phase de consultations bilatérales. Si ces consultations n'aboutissent pas, alors seulement, l'ORD peut créer un Groupe spécial et enclencher la phase quasi-juridictionnelle de la procédure (avec une possibilité d'appel pour le défendeur). La phase de mise en ouvre des recommandations du Groupe spécial ou de l'organe d'appel permanent constitue la dernière étape. Dans l'hypothèse où ces recommandations ne seraient pas exécutées par la partie concernée, la mise en œuvre de compensations ou des suspensions de concessions commerciales est prévue par l'Accord OMC. Enfin, si la partie concernée refuse les suspensions proposées, l'on entre dans l'ultime étape : celle de l'arbitrage. La procédure reste ainsi relativement longue et coûteuse (en moyens humains et financiers), mais surtout, elle s'insert dans un règlement de nature politique entre les Etats, ce qui laisse finalement une 
certaine marge de manœuvre aux pays attaqués, notamment si ceux-ci ont en mains des dossiers contre le pays plaignant. Les différends commerciaux qui ont été traités par l'ORD ces dernières années entre les deux grands partenaires commerciaux de la planète, les Etats-Unis et l'Union européenne, notamment sur la question de la banane ou sur celle (à peine entamée) de la protection des producteurs d'acier aux Etats-Unis, montrent que les conflits prennent très rapidement un caractère politique. Ainsi, les membres de l'OMC sont souvent tentés de construire patiemment des dossiers contre leurs grands partenaires commerciaux qu'ils mettent opportunément en avant lors du règlement d'un différend. Même s'il est ouvertement coupable de non-respect des accords de l'OMC, un pays mis en cause peut se servir d'autres dossiers contre le pays plaignant pour négocier avec ce dernier une solution à l'amiable dans le cadre de l'ORD.

Contrairement à bon nombre de pays en développement, la Chine dispose de moyens financiers et humains suffisants pour entrer dans ce jeu politique. Les multiples entorses aux règles de l'OMC commises par les pays développés notamment sur les subventions agricoles ou les barrières non tarifaires - qui sont un objet de conflit permanent entre les pays industrialisés et les économies en développement - ne manqueront pas d'être utilisées par la Chine en cas de conflit sur l'application des règles de l'OMC. Enfin, la Chine bénéficiera vraisemblablement, dans les années à venir, d'une relative clémence de la part des grands partenaires commerciaux concernant la transformation de son système juridique. Ceux-ci sont en effet conscients de l'immensité de la tâche à accomplir. Pascal Lamy, le commissaire européen chargé du commerce, a ainsi déclaré qu'il serait préférable de ne pas harceler la Chine durant les premières années de son accession à l'OMC.

Dans toute volonté de réforme, le comportement, soit coopératif soit rétif, des différents groupes d'acteurs (plus ou moins bien organisés) touchés par les réformes à une importance primordiale dans la réussite de la politique décidée par les pouvoirs publics ${ }^{(15)}$. Les concessions accordées par le gouvernement chinois en matière d'ouverture de son marché intérieur risquent de bousculer certaines positions de rente, tant au niveau national qu'à l'échelle provinciale. C'est justement pour cette raison que l'OMC constitue un moyen privilégié pour les autorités centrales pour passer outre certains avantages acquis ou situations privilégiées qui freinaient la dynamique interne des réformes. Pékin souhaite faire avancer certains dossiers comme l'amélioration de la gouvernance des entreprises d'Etat, la réforme du secteur bancaire, le protectionnisme régional qui fragmente le marché national ou la nécessaire sortie de marché de milliers d'entreprises inefficaces. Le gouvernement central semble suffisamment fort pour imposer des réformes douloureuses aux groupes rétifs au sein des ministères nationaux. Cependant, sa capacité à « tenir ses troupes "dans les provinces est beaucoup plus faible : les relations complexes entre le centre et les provinces en matière de fiscalité, de politique industrielle, de régulation du système financier ou d'harmonisation des systèmes de distribution en témoignent. Il est à craindre que, malgré des compensations qui restent à définir sur le plan fiscal et de la protection sociale, les provinces susceptibles de souffrir de l'ouverture restent à la traîne et fassent preuve d'une certaine inventivité en instaurant des barrières non-tarifaires.

Devant les critiques qui pourraient venir de l'étranger sur le non-respect par des gouvernements provinciaux des concessions accordées par la Chine lors de son accession à l'OMC, on peut raisonnablement penser que les autorités centrales adoptent une double attitude. D'un côté, elles afficheront un discours de fermeté destiné aux étrangers critiquant l'attitude des provinces, le tout assorti de promesses de changements ; et de l'autre, elles pencheront en faveur d'une attitude plus ou moins conciliante vis-à-vis des provinces qui souffrent de l'ouverture, attitude qui variera probablement en fonction des conséquences sociales locales de l'ouverture. Si des clauses figurant dans le protocole d'accession sont identifiées comme responsables d'une déstructuration trop rapide du tissu économique local et entraînent des conséquences sociales et politiques jugées dangereuses, l'on peut raisonnablement penser que le gouvernement central fermera les yeux et tolérera tacitement (tout en les critiquant dans le discours officiel) les pratiques discriminatoires mises en place par des provinces. Par contre, si ces conséquences sociales et politiques sont gérables, le gouvernement central adoptera une attitude plus ferme vis-à-vis des provinces en arguant de la nécessité d'appliquer les accords $\mathrm{OMC}$, ce qui lui permettra de briser certains protectionnismes locaux.

De manière générale, les pays membres de l'OMC ont su faire preuve d'une imagination débordante pour mettre en place des barrières non tarifaires ou tout simplement contrevenir aux règles de l'OMC qu'ils ont pourtant négociées. La Chine fera de même si elle estime ses intérêts économiques ou politiques menacés. Le domaine agricole, par exemple, est certainement celui où les pays industrialisés membres ont le plus de difficultés à accepter la fin négociée d'un protectionnisme qui a été à la base du développement de leur agriculture nationale. Subventions à l'exportation, barrières nontarifaires liées au contrôle sanitaire ou au respect de l'environnement pour les agricultures européennes, française notamment, sont encore largement utilisées 
pour restreindrc l'accès des produits agricoles étrangers au marché européen. Même les Etats-Unis, dont l'administration est pourtant en faveur d'une libéralisation des échanges internationaux et qui a combattu les pratiques européennes, vient de faire voter coup sur coup une loi augmentant les subventions versées aux producteurs agricoles et le relèvement des droits de douane sur l'acier afin de protéger des producteurs nationaux moribonds. Ces décisions ne manqueront pas d'inciter les pays en développement à utiliser eux aussi les armes qu'ils jugent nécessaires pour protéger leur marché intérieur. La Chine vient par exemple de décider d'imposer à son tour des droits de douane sur l'acier étranger par mesure de protection ainsi que sur l'huile de soja américaine par mesure de rétorsion ${ }^{(16)}$. S'agissant, encore une fois, des produits agricoles, la Chine s'est engagée dans son protocole d'accession à l'OMC à ne pas dépasser l'équivalent de $8,5 \%$ du total de la production agricole pour l'ensemble de ses subventions versées aux producteurs. Malgré les difficultés pour calculer l'ensemble des subventions existantes, évalué aux alentours de $2 \%$ de la production agricole, la Chine dispose d'une marge de manœuvre qu'elle pourra utiliser au gré de ses besoins. En dehors des questions agricoles, la Chine dispose également d'une multitude d'options pour réduire la portée des concessions accordées en matière d'ouverture de son marché intérieur. Dans le secteur financier, elle peut restreindre les possibilités pour les banques étrangères de se refinancer sur le marché interbancaire chinois, augmentant par là même leurs coûts vis-à-vis des banques chinoises. Sur le plan industriel, les standards, les normes sanitaires, le maintien d'un contrôle étatique sur certains réseaux de distribution ou d'importation et d'autres pratiques largement utilisées par les pays membres de l'OMC lui permettent d'amortir, si le besoin s'en fait sentir, les conséquences d'une disposition figurant dans son protocole d'accession. De manière générale, la Chine sera plus encline à utiliser ces pratiques si les pays industrialisés ne montrent pas le bon exemple comme cela s'avère être le cas aujourd'hui.

Au total, sans sous-estimer les conséquences à court et moyen terme de l'accession de la Chine à l'OMC sur l'ouverture de son marché intérieur, les phénomènes décrits ci-dessus montrent bien que ce pays dispose de plusieurs outils pour amortir et contrôler le choc liée à l'ouverture. Néanmoins, de la même manière que certaines analyses ont exagéré les conséquences potentiellement négatives des concessions accordées par la Chine pour pouvoir accéder à l'OMC, certains effets bénéfiques attendus ont parfois été surestimés, d'autres sont aujourd'hui encore difficiles à cerner tant les variables qui les influencent sont multiples et complexes.

\section{Les inconnues de l'accession à l'OMC}

\section{Les limites des modèles économétriques}

Près de 20 modèles économétriques ont été élaborés depuis 1997 pour analyser les répercussions de l'accession de la Chine à l'OMC ${ }^{(17)}$. Bien que leurs auteurs posent eux-mêmes très clairement les limites de leurs constructions mathématiques, une fois publiées, elles exercent néanmoins une forte influence sur les analyses, parfois même sur les négociations. On considère trop souvent comme acquises les prévisions des modèles alors que celles-ci devraient être envisagés tout au plus comme un cadre de réflexion sur l'agencement et l'influence à très court terme des quelques variables modélisables qui entrent en jeu dans l'accession de la Chine à l'OMC. Non seulement ces modèles souffrent de multiples limites intrinsèques, mais ils sont également loin d'avoir intégré et mesuré les répercussions multiples et complexes du processus de libéralisation.

La quasi-intégralité de ces modèles économétriques repose sur la théorie classique du commerce international dite des avantages comparatifs. D'un point de vue global, les études concluent presque unanimement à un gain positif maximum de 1,5\% de croissance du PIB par an durant la prochaine décennie (les résultats varient en fonction de la levée des barrières à l'entrée des produits textiles chinois à l'étranger). Ces modèles montrent sans surprise que l'accession de la Chine à l'OMC devrait se traduire par des gains à court et moyen terme dans les industries où la Chine dispose d'avantages comparatifs, à savoir les industries à forte intensité en travail situées dans les provinces côtières comme le textile, l'habillement ou l'assemblage des produits du secteur électronique. Par contre, les secteurs fortement protégés et dans lesquels le facteur capital (terres, technologie) est décisif, comme l'agriculture, les machines-outils ou l'automobile, devraient connaître à court terme une baisse de la production et des emplois du fait de l'arrivée de produits étrangers sur le marché intérieur. Néanmoins, selon la théorie des avantages comparatifs qui préconise une libéralisation du régime de commerce intérieur et extérieur des pays, les pertes à court terme dans ces secteurs seront compensées d'une part par des gains dans les secteurs où la Chine dispose d'avantages comparatifs, et d'autre part, par des gains d'efficacité à moyen et long terme dans les secteurs initiallement perdants. En effet, les acteurs qui opéraient à l'abri du protectionnisme vont être obligés de réagir à la concurrence des produits importés ou des entreprises étrangères produisant en Chine, soit en augmentant leur productivité, soit en se diversifiant vers d'autres produits pour lesquels ils peuvent jouir d'avantages comparatifs. De cette manière, certains modèles comme celui de Li 
Shantong et Zhai Fan prévoient la destruction de près de 9,6 millions d'emplois dans l'agriculture durant la phase transitoire ${ }^{(18)}$. Mais les mêmes auteurs indiquent dans un autre modèle que des millions d'emplois vont être créés dans l'industrie légère et les villes côtières ${ }^{(19)}$. A plus long terme, confrontés aux produits américains et européens plus compétitifs, les producteurs agricoles chinois vont réduire les sureffectifs et mécaniser leur production (augmentation de productivité), tout en se diversifiant vers d'autres produits. Les gains d'efficacité à moyen et long terme et les répercussions positives pour les consommateurs sont une des principales raisons pour préconiser l'ouverture des économies (abaissement des droits de douane et déréglementation). Selon les tenants du libre-échange, ces gains d'efficacité sont possibles non seulement dans l'industrie et l'agriculture mais également dans les services qui font l'objet de négociations depuis l'Uruguay Round.

L'objet de cet article n'est pas d'exposer les limites de la théorie des avantages comparatifs et de la libéralisation des régimes du commerce pour les pays en déve- loppement dans le cadre du droit OMC. Cependant, dans le cas chinois, ces modèles font l'impasse sur certaines variables qui renforcent les incertitudes quant aux conséquences du processus de libéralisation.

Selon ces modèles, la levée des quotas sur les textiles chinois proposée par les Etats-Unis et l'Europe et prévue dans les accords OMC, devrait assurer le secteur textile d'être le principal gagnant en termes de production et d'emplois ${ }^{(20)}$. Néanmoins, depuis plusieurs années déjà, les fabricants chinois utilisent des voies indirectes pour contourner les quotas, vendant sur des marchés tiers, notamment en Asie du sud-est, et réexportant ensuite vers les marchés américains et européens. Les gains dans l'industrie textile risquent donc d'être moins importants que ceux anticipés par les modèles économétriques. Il convient de signaler également, comme le précise Nicholas Lardy, toutes les incertitudes entourant le dynamisme du commerce international et la volonté des pays membres d'honorer leurs décisions en matière d'ouverture de leur marché intérieur aux produits chinois ${ }^{(21)}$. Bien que le commerce 
international ait connu, au cours de ces quarante dernières années, une progression continue, des périodes de ralentissement ne sont pas à exclure et entraineraient un manque à gagner pour les exportateurs chinois. Parallèlement, les pays industrialisés ont démontré par le passé leurs réticences à ouvrir leur marché intérieur malgré les engagements pris dans le cadre de l'OMC. Lors de la réunion de l'OMC à Doha, en novembrc 2001, ces barrières à l'entrée ont provoqué de multiples critiques de la part des pays en développement et ont bien failli entraîner un blocage des négociations sur la poursuite de la libéralisation du commerce. La décision du gouvernement américain de protéger ses producteurs nationaux d'acier en imposant des droits de douane au mépris des règles de l'OMC constitue un bon exemple des entorses à la liberté du commerce pourtant revendiquée par les pays industrialisés. Cette décision, ainsi que celle imposée par l'Union européenne qui cherche en réaction à protéger ses propres producteurs, coûtent cher aux producteurs d'acier chinois, et la Chine a demandé officiellement l'ouverture de négociations pour que ceux-ci soient exemptés des nouveaux droits de douane américains.

A court terme, les transferts de main d'œuvre des secteurs perdants de l'ouverture vers les secteurs gagnants seront difficiles et coûteux. Si les gains liés à l'ouverture sont concentrés dans l'industrie légère, localisée pour l'essentiel dans les provinces côtières, les dégraissages dans l'agriculture et dans les secteurs industriels perdants des provinces de l'intérieur vont accentuer les transferts de population vers les villes du littoral. La pression démographique dans les zones côtières nécessitera la mise en place d'infrastructures supplémentaires (écoles, logements, hôpitaux, routes). Celles-ci pourront certes être financées par les rentrées fiscales attendues du supplément d'activité dans ces zones, mais leur réalisation nécessitera un délai souvent assez long. Par ailleurs, la mobilité au sein du marché chinois du travail est loin d'être totale en raison des différentes barrières administratives (permis de résidence ou hukou, financement local de la protection sociale), ce qui implique que seule une partie de la population, souvent la plus jeune, va quitter sa région d'origine pour trouver un emploi. Une part de la population touchée par les dégraissages va donc rester dans les provinces de l'intérieur et devra bénéficier d'une couverture sociale minimale que les autorités ont déjà bien du mal à garantir aujourd'hui. Seul un regain de dynamisme économique des régions perdantes pourraient garantir des ajustements peu coûteux et sans heurts. Les expériences de libéralisation et d'ouverture dans les pays développés démontrent que cela est loin d'être le cas. Les restructurations sont longues et onéreuses tant sur le plan social que sur le plan économique, sans même parler des répercussions politiques qui, dans le cas de la Chine, constituent un élément important à prendre en compte.

Par ailleurs, les gains d'efficacité à moyen et long terme attendus de l'ouverture sont loin d'être certains. Dans de nombreux cas, la «main visible de l'Etat » doit supplanter la " main invisible " du marché pour permettre aux acteurs économiques nationaux de réagir en augmentant la productivité ou en diversifiant les activités pour lesquelles ils disposent d'avantages comparatifs. Dans le cas de l'agriculture, malgré les multiples effets qui risquent d'amortir le choc de l'ouverture, les paysans les plus exposés auront toutefois besoin de financements supplémentaires, d'institutions permettant l'apprentissage de nouvelles compétences et la diffusion des informations technologiques nécessaires à la mise en place de nouvelles activités. Quid des ajustements attendus dans le secteur bancaire national qui sont censés être introduits à la faveur de l'ouverture du marché chinois à la concurrence étrangère ? Les banques chinoises vont certes bénéficier d'une période plus longue que la période transitoire avant de faire face à une concurrence directe de la part des banques étrangères. Elles vont en outre jouir d'avantages de fait (cf. plus haut). Mais avant de devenir des concurrents sérieux, celles-ci vont devoir mettre en place de vastes réseaux de succursales, embaucher et former du personnel rompu aux techniques bancaires ${ }^{(22)}$. Les ajustements auxquels doit faire face le secteur bancaire chinois pour se hisser au niveau de la concurrence étrangère requièrent par conséquent un vaste programme de réformes qui vient tout juste d'être entamé. Et cet effort est tardif si on le compare à celui déployé depuis la crise asiatique de 1997 par les autres pays de la région comme la Corée du Sud, la Thailande ou la Malaisie, Etats qui, $a$ fortiori, ne pâtissaient pas de problèmes d'une ampleur comparable à ceux qu'affronte le secteur bancaire chinois aujourd'hui. Les quatre grandes banques commerciales d'Etat vont devoir être recapitalisées pour un montant qui se situe selon les prévisions plus ou moins optimistes entre $30 \%$ et $58 \%$ du PIB (soit entre 327 milliards et 632 milliards de dollars américains) ${ }^{(23)}$. Afin d'éviter l'accumulation de nouveaux prêts non performants, le gouvernement chinois va devoir mener de front la libéralisation des taux d'intérêt, le développement du marché interbancaire, l'établissement de règles prudentielles plus solides, la privatisation vraisemblable d'une bonne partie des banques commerciales d'Etat et l'amélioration considérable des systèmes de gouvernance des entreprises. Quand on connait l'état actuel du secteur bancaire chinois et que l'on mesure le travail qui a été réalisé depuis ces dix demières années, on est en droit de douter de la capacité de l'Etat chinois à mener 
à bien, sur une période aussi courte - entre 5 à 10 ans - des réformes d'une telle ampleur.

On pourrait multiplier les exemples pour montrer que, dans bon nombre de secteurs, les ajustements issus de l'ouverture seront longs et coûteux. Ils vont requérir de la part de l'Etat d'importants moyens financiers et humains ainsi qu'une refonte des institutions qui gèrent l'économie, soit tout un ensemble de mesures qui n'ont pas été prises en compte dans les modèles économétriques et dont il est bien difficile aujourd'hui de prédire l'issue.

Enfin, reste à savoir vers quel type de système économique se dirige la Chine avec l'ouverture. Selon la théorie des avantages comparatifs, la Chine devrait se spécialiser essentiellement dans les secteurs à forte intensité de main d'œuvre. Or les ambitions tout à fait légitimes des dirigeants chinois vont bien au-delà d'une telle spécialisation. La Chine aspire à monter dans la chaîne de la valeur ajoutée et à s'émanciper progressivement de la position subordonnée dans laquelle elle se trouve aujourd'hui dans la pyramide d'une sous-traitance internationale qui représente près de $55 \%$ de son commerce extérieur. La Chine aspire également à développer des firmes d'une taille mondiale disposant de fortes capacités technologiques. La question est de savoir si de telles aspirations sont compatibles avec un régime de commerce libéralisé tel que celui qui a été accepté par la Chine lors de son accession à l'OMC ${ }^{(2.4)}$.

Cette question dépasse très largement le cas de la Chine et intéresse tous les pays en développement et tout particulièrement les pays-continents comme la Russie, l'Inde ou le Brésil. Un examen de la vaste littérature sur les stratégies de rattrapage les plus couronnées de succès au cours des cinquante dernières années, montre que des pays comme le Japon, la Corée du Sud ou Taiwan, sont loin d'avoir recourus à une libéralisation de leur régime de commerce à un stade de développement économique équivalent de celui auquel se trouve la Chine actuellement ${ }^{(23)}$. Ces pays ont largement donné la priorité à leurs producteurs nationaux dans leur stratégie de développement, en contrôlant les investissements directs étrangers, en encadrant la politique de crédit, en protégeant leur marché intérieur, en favorisant l'accumulation de capacités technologiques dans des firmes nationales ou en interdisant un contrôle majoritaire de leur capital par des firmes étrangères. En choisissant la carte de l'OMC, les dirigeants chinois donnent, au contraire, la priorité aux consommateurs en leur laissant le choix entre des produits nationaux et des produits étrangers (importations ou produits de firmes étrangères implantées en Chine). L'ampleur des choix en matière de stratégie de développement et de rattrapage pour les pays en développement, il est vrai, s'est passablement restreinte sous l'impact de la globalisation de la production et de la finance ainsi qu'avec la libéralisation du commerce issue des négociations multilatérales dans le cadre de l'OMC. Les pays en développement ne peuvent plus aussi facilement pratiquer le protectionnisme pour assurer leur développement. Cependant, à la lumière des expériences asiatiques citées ci-dessus, mais également des expériences des pays développés, on est en droit de se poser de réelles questions quant à la capacité d'émancipation technologique des pays en développement, y compris de la Chine, et de leur volonté légitime de développer des firmes d'une taille globale dans le cadre de la mondialisation actuelle. Le nationalisme économique qui s'exprime par la puissance technologique de grandes firmes nationales est loin d'avoir disparu en Chine. Certes, la crise des chaebols en Corée du sud en 1997 et l'enlisement économique du Japon depuis 1990 ont considérablement terni, chez les dirigeants chinois, l'image des modèles de développement que ces deux pays véhiculaient. Mais la Chine a-t-elle pour autant renoncé à faire entrer (sans succès pour l'instant) plusieurs de ses firmes nationales dans la liste dressée par le magazine Fortune des 500 plus grandes entreprises mondiales? Les politiques de création des grands groupes chinois et de fusions dans plusieurs secteurs industriels ainsi que les mesures d'aides à la recherche et au développement indiquent que ce n'est pas le cas ${ }^{(26)}$. La Chine a-t-elle définitivement renoncé à produire une voiture chinoise alors que l'OMC semble bien l'orienter vers une situation proche de celle de la Grande Bretagne (une industrie automobile sans producteurs nationaux) ? Les protections négociées dans le cadre de l'OMC sur la production des moteurs - secteur dans lequel les firmes étrangères n'ont pas le droit d'exercer un contrôle majoritaire dans le capital - ou l'impossibilité pour les firmes étrangères d'augmenter leur participation au capital en vue de prendre un contrôle majoritaire des joint ventures dans l'automobile, montrent que les dirigeants chinois n'ont pas abandonné cet objectif ${ }^{(2 i)}$. L'on pourrait multiplier les exemples dans l'aéronautique, les télécommunications, les machines-outils, les circuits intégrés (qui en dépit d'une production croissante reste un secteur très largement dominé par les firmes étrangères). Les aspirations des dirigeants chinois n'ont rien d'extraordinaire puisqu'elles sont au cœur de la légitimité des politiques économiques des pays développés qui ont beau jeu de préconiser une libéralisation du commerce international lorsqu'ils sont dans une position de domination écrasante sur le plan financier, technologique et commercial. Quelles seront les conséquences de la nouvelle stratégie d'ouverture adoptée par la Chine sur tous ces vœux de grandeur? Comment ces 
répercussions seront-elles gérées sur le plan politique ? Autant de questions auxquelles il est bien difficile de répondre aujourd'hui et que les modèles économétriques sont bien incapables d'anticiper.

\section{Accélération et modification}

\section{de la stratégie de réforme}

Dans un entretien récent, Sir Leon Brittan, ancien commissaire européen au commerce et qui, à ce titre, participa aux négociations d'accession de la Chine à l'OMC, confiait qu'au cours d'un entretien avec le Premier ministre Zhu Rongji, celui-ci lui aurait déclaré que les concessions demandées à la Chine étaient des réformes qui, de toutes les manières, auraient été mises en place même si la Chine n'était pas entrée dans l'OMC ${ }^{(28)}$. Cette déclaration d'un des principaux artisans de la politique économique de l'après-Deng Xiaoping n'était pas uniquement destinée à obtenir des concessions supplémentaires de la part des grands partenaires commerciaux de la Chine. Elle reflétait aussi la décision, indépendante du résultat des négociations $\mathrm{OMC}$, d'utiliser l'ouverture et la déréglementation du marché intérieur comme l'un des instruments privilégiés du vaste chantier de réformes à venir.

Depuis le milieu des années 1990, les dirigeants chinois ont pris très largement conscience du fait que les problèmes auxquels fait face l'économie chinoise s'alimentent les uns les autres et risquent, si rien n'est entrepris, de créer une crise systémique du type de celles traversées par les pays asiatiques en 1997 avec, $a$ fortiori, le spectre d'une déstabilisation politique à l'image de l'Indonésie. Rien n'illustre mieux cette interdépendance des problèmes que le développement rapide des prêts non performants dans le secteur bancaire. Ce phénomène est en effet l'expression macro-économique d'une mauvaise gouvernance tant à l'échelle des cntreprises qu'à l'échelle de l'Etat. La résolution de ce problème est urgente car il en va de la santé fiscale future (l'Etat doit s'endetter pour recapitaliser les banques) et de manière plus générale d'une utilisation plus efficace des ressources financières nationales. Pour résoudre ce problème, l'Etat doit néanmoins intervenir sur tous les fronts : celui de la gouvernance des entreprises en améliorant des dossiers épineux allant de la transparence des activités des conseils d'administration jusqu'à la possibilité pour les actionnaires minoritaires d'engager une action en justice contre des chefs d'entreprises malveillants, en passant par le rôle omniprésent du Parti communiste dans le processus décisionnel des entreprises publiques. L'Etat doit également réformer le système bancaire en améliorant les règles prudentielles, en dépolitisant les circuits décisionnels, et en développant de meilleures compétences dans la gestion du risque. De la même manière, la réduction des prêts non performants passe par des restructurations qui impliquent des faillites en nombre important, ce qui demande à son tour une amélioration urgente du système de protection sociale. Les dirigeants chinois doivent ainsi intervenir simultanément sur tous les fronts et ceci sans position de repli s'ils ne veulent pas risquer de voir la situation leur échapper.

Dans un tel contexte, se pose bien évidemment la question des ressorts et incitations intermes en matière de réformes. Il fallait trouver un instrument qui permette de galvaniser les énergies tout en créant une irréversibilité sur le plan du calendrier et du contenu des réformes. Par ailleurs, certaines réformes demandent des sacrifices à certains groupes d'acteurs, aux provinces et aux ministères. Pour briser des situations de rente établies durant les années 1980 et la première moitié des années 1990, il fallait trouver un instrument qui échappe à tout détournement ou à toute dénaturation par une coalition d'intérêts hostiles aux réformes. On comprend mieux, dans ce contexte, la remarque de Zhu Rongji et son action déterminante dans la conclusion des négociations sur l'adhésion de la Chine à l'OMC (le voyage aux Etats-Unis en avril 1999, ses interventions dans la phase finale). L'ouverture et la déréglementation ont non seulement été placées au cœur du processus des réformes, mais elles ont été négociées dans le cadre d'un accord international multilatéral, ce qui leur donne tous les attributs et qualités nécessaires pour devenir un catalyseur essentiel des réformes. Laccession de la Chine à l'OMC marque en quelque sorte une rupture définitive avec l'héritage économique de Deng Xiaoping qui, à tort ou a raison, avait privilégié une approche partielle (en terme de problèmes à résoudre) et graduelle (en terme de rythme). L'accession de la Chine à l'OMC est avant tout un instrument interne de poursuite des réformes sur un mode et à un rythme inédits, ce qui représente un nouveau pari pour le pouvoir chinois et dont il est difficile de prévoir l'issue. Il sera également difficile de discerner ce qui relève désormais de la poursuite des réformes ou de l'application des concessions accordées par la Chine pour son accession à l'OMC. L'OMC risque, dans ce cadre, de cristalliser les attentes, les critiques et les mécontentements quand la poursuite des réformes débouchera sur des problèmes.

$\mathrm{A}^{\mathrm{N}}$ conclusion, l'accession de la Chine à l'OMC donne Sune impulsion qui était devenue de plus en plus difficile à générer sur le plan interne pour conduire des réformes douloureuses et importantes. Réformer en invoquant la pression extérieure tout en gardant la possibilité en cas d'échec ou de difficultés de faire porter la responsabilité à l'étranger, est désormais un grand clas- 
sique en matière de modernisation d'une économie. Avec l'épuisement de la dynamique politique interne issue de l'ère Deng Xiaoping, les dirigeants chinois actuels avaient particulièrement besoin de ce motif pour passer outre les avantages acquis des différentes catégories touchées par les réformes à venir sans accorder de contre-parties excessives qui destabiliseraient l'équilibre financier du pays.

Si l'on ajoute les avantages escomptés de l'accession en matière d'exportation, d'investissements directs étrangers, de libéralisation du marché intérieur et de potentiels gains d'efficacité liés à la libéralisation, on peut être relativement optimiste sur le pari économique qu'ont fait les dirigeants chinois. En outre, les autorités chinoises bénéficient de multiples garde-fous qui les protègent contre un choc trop violent lié à l'ouverture et qui leur laisse une latitude non négligeable dans le rythme et l'étendue des adaptations à mettre en œuvre pour gérer au mieux l'ouverture du marché intérieur.

Il convient toutefois de tempérer cet optimisme, car plusieurs facteurs importants, aujourd'hui difficilement quantifiables, incitent à la prudence et à l'humilité dans la compréhension des répercussions de l'accession de la Chine à l'OMC : la stabilité d'un environnement international favorable capable d'absorber les exportations chinoises ; le coût social des ajustements structurels de l'emploi dans l'industrie et surtout dans l'agriculture ; la capacité des firmes chinoises à affronter une concurrence étrangère sur leur marché intérieur ; et enfin les difficultés à mener de front des réformes de grande ampleur, comme celles du secteur financier et du secteur public. Tous ces facteurs sont difficilement "modélisables". La théorie des avantages comparatifs sur laquelle repose l'essentiel des modèles économétriques qui ont tenté de mesurer les conséquences économiques de l'accession de la Chine à l'OMC est largement muette sur ces questions, et de ce fait bien incapable de mesurer leur éventuel pouvoir de nuisance. En fait, derrière l'évolution positive de ces différentes inconnues, l'enjeu est tout simplement le passage réussi de la demière étape, peut-être la plus périlleuse et la plus difficile, celle de la transition vers une économie de marché que la Chine a entamée voilà presque un quart de siècle. $\$$

1. Pour un résumé détaillé des engagements pris par la Chine pour son accession à l'OMC voir l'annexe I, p. 749 de OCDE, China in the World Economy. The Domestic Policy Challenges, Paris, OCDE, 2002, 813 p.

2. Andrzej Kwiecinski, "Implications for the Rural Economy", OCDE, China in the World Economy. The Domestic Policy Challenges, Paris, OCDE, 2002, pp. 105-124.

3. Pour une revue détaillée de l'accord voir p. $77-78$ de Nicholas $R$. Lardy, Integrating China into Global Economy, Washington D.C., Brooking Institution Press, 2002, 244 p.
4. N. Lardy, op. cit., 2002, p. 78.

5. A. Kwiecinski, op. cit., 2002

6. Voir annexe II, p. 761 in OCDE, Chino in the World Economy, op. cit.

7. Interviews auprès des dirigeants de Citroën-Dongfeng à Wuhan, août 2001.

8. South China Morning Post, 22 novembre 1998.

9. South China Morning Post, 22 novembre 1998.

10. Asian Wall Street Journal, 5 mars 1999.

11. Cité par le South China Morning Post, 11 novembre 1998.

12. N. Lardy, op. cit., 2002.

13. Voir dans la première partie du dossier Chine OMC, l'article de Leila Choukroune, "L'Etat de droit par l'internationalisation, objectif des réformes ", Perspectives Chinoises, $n^{\circ} 69$, janvier-février 2002, pp. $7-22$.

14. Instance de règlements des litiges commerciaux au sein de l'OMC.

15. Andrei Shleifer et Daniel Treisman, Without a map. Political Tactics and Economical Reform in Russia, Cambridge, Massachusetts, MIT, 2001, $223 \mathrm{p}$.

16. La Chine a décidé d'imposer, le 24 mai 2002, des droits de douane pendant 180 jours allant de $7 \%$ à $26 \%$ selon les catégories considérées d'acier importé et pourrait augmenter les droits de douane sur l'huile de soja américaine jusqu'aux environs de 24\%. South China Morning Post, 23 mai 2002.

17. Voir annexe II p. 761 in OCDE, op.cit., 2002.

18. Li Shantong et Zhai Fan, " The Implications of Acession to WTO on China's Economy ", Third Annual Conference on Global Economic Analysis, Melbourne, Australie, 27-30 juin, 2000.

19. Li Shantong et Zhai Fan, "China's WTO Accession and Implications for National and Provincial Economies ", Conférence au CEPII, Paris, 2001.

20. Voir annexe II p. 761 in OCDE, op.cit. 2002, pour les différences entre les modèles qui prennent en compte la levée des quotas sur le textile.

21. N. Lardy, op. cit., 2002, p. 78.

22. Voir John Thompson \& Charles Pigott, "Developing the Financial System and Financial Regulatory Policies", OCDE, China in the World Economy, op. cit. Voir également John D. Langlois, JR., "The WTO and China's Financial System ", The China Quarterly, septembre 2001, pp. 610-655.

23. John Thompson et Charles Pigott, op. cit., OCDE, 2002.

24. Jean-François Huchet et Xavier Richet, "Between Bureaucracy and Market : Chinese Industrial Groups in Search of New Forms of Corporate Governance ", Journal of Post-Communist Economies, vol. 14, n², 2002, pp. 170-201.

25. Voir l'excellent article de K.S. Jomo, "Southeast Asia: From Miracle to Debacle ", in Luigi Tomba éd., East Asian Capitalism: Conflicts, Growth and Crisis, Annali della Fondazione Giangiacomo Feltrinelli, $n^{\circ}$ XXXVI, Milan, Feltrinelli, 2002, pp. XLIII, 524 ; Peter Evans, Embedded Autonomy. States \& Industrial Transformation, Princeton, Princeton University Press, 1995, 323 p. ; World Bank, The East Asian Miracle : Economic Growth and Public Policy, World Bank et Oxford University Press, Londres, 1997.

26. Jean-François Huchet, "Industry Reorganisation and Restructuring: Prospects, Problems, and Policy Priorities ", in China in the World Economy. The Domestic Policy Challenges, op. cit., pp. 161-192.

27. Voir l'article d'Eric Harwit, "The Impact of WTO Membership on the Automobile Industry in China ", The China Quarterly, septembre 2001, pp. 655-670.

28. Far Eastern Economic Review, 23 mai 2002. 